\title{
In-vitro Antimicrobial Susceptibility Pattern of Isolates from Urine in Butembo, Democratic Republic of the Congo
}

\author{
Bunduki $\mathrm{GK}^{1^{*}}$, Kibendelwa $\mathrm{ZT}^{2}$ and Nzanzu $\mathrm{AK}^{3}$ \\ ${ }^{1}$ Faculty of Medicine, Université Catholique du Graben, Butembo, Democratic Republic of the Congo \\ ${ }^{2}$ Faculty of Medicine, Department of Internal Medicine, Université de Kisangani, Democratic Republic of the Congo \\ ${ }^{3}$ Matanda Hospital, Department of Internal Medicine, Butembo, Democratic Republic of the Congo
}

"Corresponding author: Gabriel Kambale Bunduki, MD, Faculty of Medicine, Université Catholique du Graben, Butembo, Democratic Republic of the Congo, Tel: +243-99-243-14 47; E-mail: gabriel.bunduki@gmail.com

Received date: November 25, 2017; Accepted date: December 11, 2017; Published date: December 12, 2017

Copyright: (c) 2017 Bunduki GK, et al. This is an open-access article distributed under the terms of the Creative Commons Attribution License, which permits unrestricted use, distribution, and reproduction in any medium, provided the original author and source are credited.

\begin{abstract}
Objective: This study aimed to determine the isolates from urine cultures at patients suspected with urinary tract infection and their in-vitro susceptibility pattern to antimicrobials at the Central Laboratory of Research of the "Université Catholique du Graben" (UCG) in Butembo

Methodology: This was a cross-sectional study adopting a descriptive approach, conducted from January, 2015 to December, 2016. Six hundred and seventeen patients were screened. Freshly voided mid- stream urine sample were taken according to standard method. Isolation of the microbial agents of urinary tract infections was done on blood agar and MacConkey agar media. The culture was repeated in contrast with significant bacteria and urinalysis if the isolate at the first culture is known non-pathogenic. The antimicrobial susceptibility testing was done by using disk diffusion (Kirby Bauer's) technique.
\end{abstract}

Results: Out of the 617 patients screened, the culture was positive in $77.3 \%$ (477 cases). The most isolated bacteria from urine culture were Staphylococcus aureus (47\%), Streptococcus spp (12\%), Escherischia coli (10.9\%), Moraxella spp $(7.5 \%)$ and Bacillus spp (2.3\%). Antibiotics which were sensible to three or more isolates were ciprofloxacin, cefuroxime, cefotaxime and vancomycin. It was also observed that all the bacterial species have a Multiple Antibiotic Resistance Index greater than 0.2.

Conclusion: Continuous monitoring of antimicrobial susceptibility pattern of bacterial isolates implicated in urinary tract infections is needed seeing the high Multiple Antibiotics Resistance Indices of all samples isolates. And this should be prior to antibiotic prescription in order to ensure an optimal, desired and cost-effectiveness treatment. For empiric treatment of urinary tract infections in Butembo, we suggested ciprofloxacin, cefuroxime and cefotaxime as the first line antibiotics of choice.

Keywords: Antibiotics; Democratic Republic of the Congo; Resistance; Urinary tract infections

\section{Introduction}

The growth of resistant bacteria to commonly used antimicrobials became a concern of the worldwide public health $[1,2]$. The expansion of antimicrobial resistance (AMR) does not only entail an increase of morbidity and mortality but constitutes also to a heavy economic burden. The health systems in countries with weak income and that fight against the lack of financing and the faltering institutions are particularly hit by AMR [3-5].

The rational use of antimicrobials has an incontestable advantage, but physicians and the public use them irrationally [6,7]. In developing countries, the development and propagation of AMR are increasing because of irrational use of antimicrobials, the maladjusted or nonexistent programs fighting against infections, bad quality drugs, weakness of laboratory capacity and deficiencies of surveillance and insufficiencies of the regulation on the use of antimicrobials [3]. In the Democratic Republic of the Congo (DRC), the availability of antimicrobials in free-market that means even without medical prescription is one of the reasons aggravating the expansion of the AMR [4]. The uncontrolled and irrational use of folk medicine is also among the reasons [8].

Multidrug resistant (MDR) pathogens propagate not only locally, regionally but also globally, with new pathogens introduced recently that spread quickly at the susceptible hosts [9]. So, surveillance data needed to be gathered and of sentries centers selected. The local epidemiology of AMR should constantly be updated to detect some changes in the causal pathogens and their sensibility to different antimicrobials.

The urinary tract infections (UTI) constitute the second motive of consultation and prescription of antimicrobials in the physician's office and in emergencies services in France [10]. While the UTI are well documented worldwide [11-13], there are not any national reports currently on the prevalence of UTI in DRC. Nevertheless, there are some regional reports. Thus, Irenge et al. [14] found in the South-Kivu a rate of AMR of $21.1 \%$ among hospitalized patients and $8.4 \%$ among out-patients. The bacteria more met were Escherichia coli, Klebsiella spp. and Enterobacter spp. 
In Butembo, the bacteria responsible for the UTI are mainly the Staphylococcus aureus (57.2\%), Escherischia coli (4.71\%), Streptococcus (4.13\%) and Moraxella (2.73\%) [15], but no survey has been done for determining their sensitivity to antimicrobials.

The knowledge of bacteria causing the UTI and their sensitivity to antimicrobials is necessary to assure an appropriate treatment. Therefore, this study aimed to determine isolates from urine of patients with UTI and their sensitivity to antimicrobials at patients attending the Central Laboratory of Research of the "Université Catholique du Graben" (UCG) in Butembo.

\section{Material and Methods}

\section{Study design}

This was a cross-sectional study adopting a descriptive approach, conducted from January 2015 to December 2016 in the department of microbiology at the Central Laboratory of Research of the "Université Catholique du Graben" (UCG) in Butembo.

\section{Population and sample size}

The population study was constituted by patients with suspicion of urinary tract infection (UTI) attending the Central Laboratory of Research of the UCG for urine culture. Patients living out of Butembo, those whose urine samples were sent to the laboratory by hospitals, those who were taking antimicrobials, and those who did not consent to the study were excluded from this study. Furthermore, cultures showing fungal growth, mixed growth and those not conclusive in the laboratory, irrespective of reason, were excluded.

The sample size was exhaustive including all patients who met the inclusion criteria. Convenience selection of urine culture reports was employed as a sampling method. Thus, 617 patients were retained for this study.

\section{Specimen collection}

Freshly clean-voided mid-stream urine sample were taken according to standard method [16]. It was collected in a sterile wide mouth container. All the urine samples were processed within one hour after the collection for aerobic bacterial culture. If delayed, samples were refrigerated and processed within 4-6 hours.

\section{Specimen processing}

Urine specimen was cultured for isolation of the microbial agents of UTI on blood agar and MacConkey agar media. A measured amount of urine, using calibrated loop method was inoculated to nutrient agar medium for colony count. Equal or more than $10^{5} \mathrm{CFU} / \mathrm{ml}$ of a single potential pathogen or for each of two potential pathogens interpreted as positive UTI and a result of $10^{2}-10^{4} \mathrm{CFU} / \mathrm{ml}$ was repeated. A less than $10^{2} \mathrm{CFU} / \mathrm{ml}$ was interpreted as negative UTI. An organism known as normal flora were considered as pathogen if after the first culture, the repeated second culture, where sample were taken carefully, isolated the same organism, with a contrast with significant bacteriuria and in conjunction with the urinalysis results. All the bacteria isolated from urine in this study were identified using conventional biochemical tests [17].

\section{Antimicrobial susceptibility study}

The antimicrobial susceptibility testing was done on Mueller-Hinton Agar using disk diffusion (Kirby Bauer's) technique. This method was done according to Clinical Laboratory Standard Institute guidelines to determine susceptibility of UTI agents. The antibiotic disks comprised, oxacilline $(1 \mu \mathrm{g})$, amoxicillin-clavulanic acid (augmentin) $(20+10 \mu \mathrm{g})$, cefuroxime $(30 \mu \mathrm{g})$, cefotaxime $(30 \mu \mathrm{g})$, ceftriaxone $(30 \mu \mathrm{g})$, meropenem $(10 \mu \mathrm{g})$, vancomycin $(30 \mu \mathrm{g})$, gentamicin $(10 \mu \mathrm{g})$, kanamycin $(30 \mu \mathrm{g})$, neomycin $(30 \mu \mathrm{g})$, spectinomycin $(100 \mu \mathrm{g})$, chloramphenicol $(30 \mu \mathrm{g})$, doxycycline $(30 \mu \mathrm{g})$, erythromycin $(15 \mu \mathrm{g})$, azithromycin $(15 \mu \mathrm{g})$, clarithromycin $(15 \mu \mathrm{g})$, clindamycin $(2 \mu \mathrm{g})$, nalidixic acid $(30 \mu \mathrm{g})$, ciprofloxacin $(5 \mu \mathrm{g})$ and levofloxacin $(5 \mu \mathrm{g})$ [18]. Microbial with intermediate susceptibility was considered as resistant for data analysis.

\section{The Multiple Antibiotic Resistance Indices (MARI)}

The MARI calculation was done by dividing the number of antibiotics to which a microorganism is resistant by the total number of antibiotics to which the organism was subjected to. Bacteria with a MARI greater than 0.20 implies that the resistance strains of such bacteria are originated from an environment where several antibiotics are used or misused.

\section{Data analysis}

We used the software WHONET 5.6 for data analysis. Results were expressed as counts and percentages. Association of variables was analysed by using odds ratio (OR), with a confidence interval of $95 \%$ (95\% CI). We considered associations as significant when OR were greater than 1 with a P-value of less than 0.05 .

\section{Ethical consideration}

Ethical clearance (CE-UCG/FACMED/001-2015) was obtained from the local research ethics committee of the Faculty of Medicine at the "Universite Catholique du Graben". Patients were explained and adequately informed on the purpose of the study and were assured of privacy and confidentiality. A consent form was signed by the patient who accepted participation to the study. For patients under 18 years, the consent was signed by their parents or guardians.

\section{Results}

Out of the 617 patients screened, the urine culture is positive in $77.3 \%$ (477 cases). The urine culture proved to be positive in $63.7 \%$ of females and the age group of $26-35$ is the more touched (44.2\%) but statistically the age group of 6-15 is more touched (OR=0.3, IC: $0.1-0.7$ and $\mathrm{P}=0.003$ ) (Table 1 ). The Figure 1 shows that Gram positive bacteria are isolated in $62.9 \%$ and Gram negative bacteria in $37.1 \%$ (Figure 1). Following their frequency, the most isolated bacteria from urine culture are Staphylococcus aureus (47\%), Streptococcus spp (12\%), Escherischia coli (10.9\%), Moraxella spp (7.5\%) and Bacillus spp (2.3\%) (Table 2). The Table 3 shows that Staphylococcus aureus was resistant to all antibiotics tested except for cefuroxime, clindamycin, ciprofloxacin levofloxacin, doxycycline, neomycin and vancomycin to which it was sensible. Streptococcus spp. was resistant to all antibiotics tested except for ceftriaxone, cefotaxime, ciprofloxacin and vancomycin. Escherischia coli were only sensitive to ceftriaxone, kanamycin, spectinomycin, azythromycin and ciprofloxacin. The MARI of all bacterial isolates was greater than 0.20 (Table 4). 
Citation: Bunduki GK, Kibendelwa ZT, Nzanzu AK (2017) In-vitro Antimicrobial Susceptibility Pattern of Isolates from Urine in Butembo, Democratic Republic of the Congo. J Antimicrob Agents 3: 154. doi:10.4172/2472-1212.1000154

Page 3 of 7

\begin{tabular}{|c|c|c|c|c|c|}
\hline \multirow{2}{*}{ Variables } & \multicolumn{2}{|c|}{ Results of urine culture } & \multirow[t]{2}{*}{ OR } & \multirow{2}{*}{$95 \%$ IC } & \multirow{2}{*}{ P-value } \\
\hline & Positive, n (\%) & Negative, $n(\%)$ & & & \\
\hline \multicolumn{6}{|l|}{ Sex } \\
\hline Female & $304(63.7)$ & $83(59.3)$ & 1.2 & $0.8-1.8$ & \multirow{2}{*}{0.339} \\
\hline Masculine & $173(36.3)$ & $57(40.7)$ & 0.8 & $0.6-1.2$ & \\
\hline Total & $477(100)$ & $140(100)$ & - & - & - \\
\hline \multicolumn{6}{|c|}{ Age (years) } \\
\hline$\leq 5$ & $13(2.7)$ & $3(2.1)$ & 1.3 & $0.3-5.74$ & 0.703 \\
\hline 6 & $8(1.7)$ & $9(6.4)$ & 0.3 & $0.1-0.7$ & 0.003 \\
\hline $16-25$ & $67(14.1)$ & $19(13.6)$ & 1 & $0.6-1.9$ & 0.887 \\
\hline $26-35$ & $211(44.2)$ & $65(46.5)$ & 0.9 & $0.6-1.4$ & 0.646 \\
\hline $36-45$ & $113(23.7)$ & $28(20.0)$ & 1.2 & $0.8-2.0$ & 0.361 \\
\hline$\geq 46$ & $65(13.6)$ & $16(11.4)$ & 1.2 & $0.7-2.3$ & 0.498 \\
\hline Total & $477(100)$ & $140(100)$ & - & - & - \\
\hline
\end{tabular}

Table 1: Socio-demographic characteristics of patients.

\begin{tabular}{|c|c|}
\hline Bacterial isolates & n (\%) \\
\hline Staphylococcus aureus & $224(47,0)$ \\
\hline Streptococcus spp & $57(12,0)$ \\
\hline Escherichia coli & $52(10,9)$ \\
\hline Moraxella spp & $36(7,5)$ \\
\hline Bacillus spp & $11(2,3)$ \\
\hline Erwina spp & $9(1,9)$ \\
\hline Listeria spp & $8(1,7)$ \\
\hline Edwardsiella hoshinae & $6(1,3)$ \\
\hline Yersinia frederiksenii & $6(1,3)$ \\
\hline Pneuteura spp & $6(1,3)$ \\
\hline Cedecea lopagei & $5(1,1)$ \\
\hline Flavobacterium odoratum & $5(1,1)$ \\
\hline Aeromonas hydrophila & $4(0,8)$ \\
\hline Klebsiella pneumoniae & $4(0,8)$ \\
\hline Ledercia adercaboxylata & $3(0,6)$ \\
\hline Salmonella spp & $3(0,6)$ \\
\hline Citrobacter freundii & $3(0,6)$ \\
\hline Pseudomonas spp & $2(0,4)$ \\
\hline Klebsiella rhinoscleromatis & $2(0,4)$ \\
\hline
\end{tabular}


Citation: Bunduki GK, Kibendelwa ZT, Nzanzu AK (2017) In-vitro Antimicrobial Susceptibility Pattern of Isolates from Urine in Butembo, Democratic Republic of the Congo. J Antimicrob Agents 3: 154. doi:10.4172/2472-1212.1000154

Page 4 of 7

\begin{tabular}{|l|l|}
\hline Budvicia aquatica & \\
\hline Escherichia vulneris $(0,4)$ \\
\hline Neisseria gonorrheae & \\
\hline Piesiomonas shigeloides & \\
\hline Cirobacter diviersys & \\
\hline Others ${ }^{*}(0,4)$ \\
\hline Total, $\mathrm{n}(\%)$ & \\
\hline $\begin{array}{l}{ }^{*} \text { Others : Enterobacter agglomeran, Enterobacter amorigenus, Enterobacter cloacea, Escherichia fergusonii, Ewingili americana, Pasteurella aerogenes, Proteus } \\
\text { penneri, Pseudomonas aeruginosa, Rnanella adualis, Salmonella typhi, Serratia ficana, Serratia liquefacens, Serratia marcescens, Vibrio hollisae, Yersinia } \\
\text { enterocolitica, Cedecea neteri, Klebsiella ozaenae, Moellerella }\end{array}$ \\
\hline
\end{tabular}

Table 2: Incidence of bacterial isolates from urine samples of the patients.

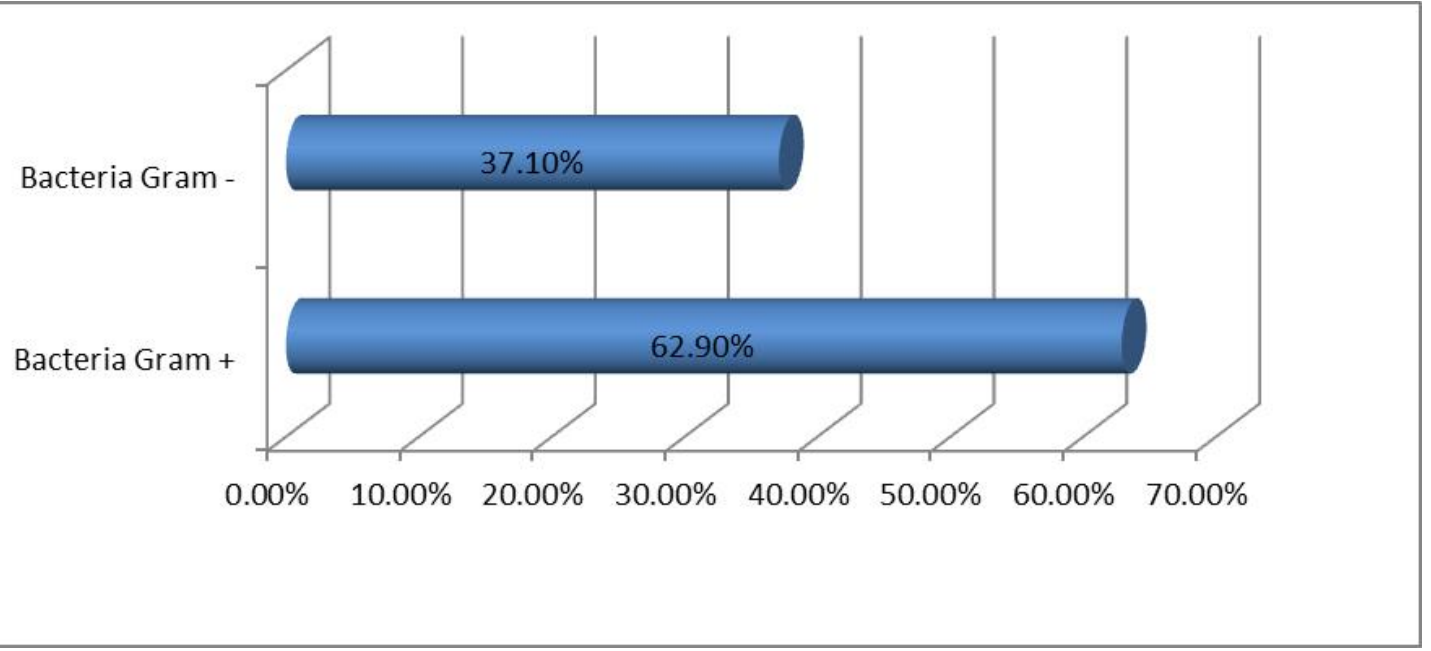

Figure 1: Repartition of bacteria according to their Gram Staining.

\begin{tabular}{|l|l|l|l|l|l|l|l|l|l|l|}
\hline \multirow{2}{*}{ Antibiotics } & \multicolumn{3}{|l|}{ S. aureus } & \multicolumn{3}{l|}{ Streptococcus spp } & \multicolumn{2}{l|}{ E. coli } & \multicolumn{3}{l|}{ Moraxella spp } & \multicolumn{2}{l|}{ Bacillus spp } \\
\cline { 2 - 12 } & $\mathrm{S}$ & $\mathrm{R}$ & $\mathrm{S}$ & $\mathrm{R}$ & $\mathrm{S}$ & $\mathrm{R}$ & $\mathrm{S}$ & $\mathrm{R}$ & $\mathrm{S}$ & $\mathrm{R}$ \\
\hline Augmentin & $31(18.4)$ & $137(81.6)$ & $17(40.5)$ & $25(59.5)$ & $2(5.9)$ & $32(94.1)$ & $5(25.0)$ & $15(75.0)$ & $1(10.0)$ & $9(90.0)$ \\
\hline Oxacilline & $2(3.0)$ & $64(97.0)$ & $2(14.3)$ & $12(85.7)$ & $0(0.0)$ & $3(100)$ & $2(50.0)$ & $2(50.0)$ & $0(0.0)$ & $1(100)$ \\
\hline Meropeneme & $14(40.0)$ & $21(60.0)$ & $2(25.0)$ & $6(75.0)$ & $8(44.4)$ & $10(55.6)$ & $3(75.0)$ & $1(25.0)$ & $0(0.0)$ & $1(100)$ \\
\hline Cefuroxime & $28(63.6)$ & $16(36.4)$ & $6(40.0)$ & $9(60.0)$ & $4(33.3)$ & $8(66.7)$ & $3(50.0)$ & $3(50.0)$ & $2(100)$ & $0(0.0)$ \\
\hline Ceftriaxone & $62(37.6)$ & $103(62.4)$ & $21(56.8)$ & $16(43.2)$ & $21(52.5)$ & $19(47.5)$ & $9(42.9)$ & $12(57.1)$ & $3(33.3)$ & $6(66.7)$ \\
\hline Cefotaxime & $9(29.0)$ & $22(71.0)$ & $3(60.0)$ & $2(40.0)$ & $4(44.4)$ & $5(55.6)$ & $6(60.0)$ & $4(40.0)$ & $1(100)$ & $0(0.0)$ \\
\hline Vancomycin & $36(87.8)$ & $5(12.2)$ & $8(80.0)$ & $2(20.0)$ & $0(0.0)$ & $1(100)$ & $1(50.0)$ & $1(50.0)$ & $1(100)$ & $0(0.0)$ \\
\hline Gentamycin & $65(49.2)$ & $67(50.8)$ & $17(37.8)$ & $28(62.2)$ & $17(44.7)$ & $21(55.3)$ & $19(61.3)$ & $12(38.7)$ & $4(100)$ & $0(0.0)$ \\
\hline Spectinomycin & $9(20.9)$ & $34(79.1)$ & $1(8.3)$ & $11(91.7)$ & $12(80.0)$ & $3(20.0)$ & $1(33.3)$ & $2(66.7)$ & $1(100)$ & $0(0.0)$ \\
\hline
\end{tabular}


Citation: Bunduki GK, Kibendelwa ZT, Nzanzu AK (2017) In-vitro Antimicrobial Susceptibility Pattern of Isolates from Urine in Butembo,

Page 5 of 7

\begin{tabular}{|c|c|c|c|c|c|c|c|c|c|c|}
\hline Neomycin & $6(50.0)$ & $6(50.0)$ & $0(0.0)$ & $3(100)$ & $2(40.0)$ & $3(60.0)$ & $0(0.0)$ & $1(100)$ & ND & ND \\
\hline Kanamycin & $0(0.0)$ & $5(100)$ & $0(0.0)$ & $1(100)$ & $1(100)$ & $0(0.0)$ & ND & ND & ND & ND \\
\hline Chloramphenicol & $11(43.8)$ & $27(56.2)$ & $4(33.3)$ & $8(66.7)$ & $2(33.3)$ & $4(66.7)$ & $3(50.0)$ & $3(50.0)$ & $1(33.3)$ & $2(66.7)$ \\
\hline Doxycycline & 109 (52.9) & $97(47.1)$ & $24(49.0)$ & $25(51.0)$ & $10(27.8)$ & $26(72.2)$ & $26(78.8)$ & $7(21.2)$ & $3(37.5)$ & $5(62.5)$ \\
\hline Clindamycin & $135(65.2)$ & $72(34.8)$ & $15(27.3)$ & $40(72.7)$ & $3(6.4)$ & 44 (93.6) & $10(30.3)$ & $23(69.7)$ & $3(30.0)$ & $7(70.0)$ \\
\hline Erythromycin & $50(32.7)$ & $103(67.3)$ & $10(25.0)$ & $30(75.0)$ & $2(5.0)$ & $38(95.0)$ & $5(21.7)$ & $23(78.3)$ & $1(11.1)$ & $8(88.9)$ \\
\hline Azithromycin & $0(0,0)$ & $47(100)$ & $1(7.1)$ & $13(92.9)$ & $8(50.0)$ & $8(50.0)$ & $2(50.0)$ & $2(50.0)$ & $0(0.0)$ & $1(100)$ \\
\hline Clarithromycin & $7(15.2)$ & $39(84.8)$ & $2(14.3)$ & $12(85.7)$ & $1(6.7)$ & $14(93.3)$ & $1(50.0)$ & $1(50.0)$ & ND & ND \\
\hline Ciprofloxacin & $148(64.3)$ & $82(35.7)$ & $36(63.2)$ & $21(36.8)$ & $26(52.0)$ & $24(48.0)$ & $25(67.6)$ & $12(32.4)$ & $6(54.5)$ & $5(45.5)$ \\
\hline Levofloxacin & $23(53.5)$ & $20(46.5)$ & $3(23.1)$ & $10(76.9)$ & $3(20.0)$ & $12(80.0)$ & $2(40.0)$ & $3(60.0)$ & $1(100)$ & $0(0.0)$ \\
\hline Nalidixic acid & $4(8.0)$ & $46(92.0)$ & $0(0.0)$ & $7(100)$ & $8(22.9)$ & $27(77.1)$ & $22(61.1)$ & $14(38.9)$ & $0(0.0)$ & $2(100)$ \\
\hline
\end{tabular}

Table 3: Antimicrobial susceptibility pattern of the bacterial isolates from urine specimens.

\begin{tabular}{|l|l|l|l|}
\hline Isolates & MARI & $\begin{array}{l}\text { Total number of } \\
\text { antibiotic tested }\end{array}$ & Antibiotics to which the isolates are resistant \\
\hline S. aureus & 0.65 & 20 & AMC, OXA, CRO, CTX, GEN, SPT, KAN, CHL, ERY, AZM, CLR, NAL and MEM \\
\hline Streptococcus spp & 0.8 & 20 & AMC, OXA, CXM, GEN, SPT, NEO, KAN, CHL, DOX, CLI, ERY, AZM, CLR, LVX, NAL and MEM \\
\hline E. coli & 0.75 & 20 & AMC, OXA, CTX, CXM, GEN, NEO, CHL, DOX, CLI, ERY, CLR, LVX, NAL, MEM and VAN \\
\hline Moraxella spp & 0.37 & 19 & AMC, CRO, SPT, NEO, CLI, ERY and LVX, \\
\hline Bacillus spp & 0.59 & 17 & AMC, OXA, CRO, CHL, DOX, CLI, ERY, AZM, NAL and MEM \\
\hline $\begin{array}{l}\text { AMC: Augmentin; OXA: Oxacilline; CRO: Ceftriaxone; CTX: Cefotaxime; CXM: Cefuroxime; GEN: Gentamycin; SPT: Spectinomycin; NEO: Neomycin; KAN : } \\
\text { Kanamycin; CHL: Chloramphenicol; DOX: Doxycycline; CLI: Clindamycin; ERY: Erythromycin; AZM: Azithromycin;CLR: Clarithromycin; CIP: Ciprofloxacin; LVX: } \\
\text { Levofloxacin; NAL: Nalidixic Acid (Negram); MEM: Meropeneme; VAN: Vancomycin }\end{array}$ &
\end{tabular}

Table 4: Multiple Antibiotic Resistance Indices (MARI) of the bacterial isolates.

\section{Discussion}

Urine cultures were positive in $63.7 \%$ of females and $36.3 \%$ of males as shown in Table 1 . This result shows that, females are more vulnerable for the urinary tract infections (UTI) than males. These results are close to the ones of Bentroki et al. in their survey on the antibiotics resistance of isolated stumps of communal urinary infections between 2007 and 2011 in Guelma (Algeria). They had observed predominance of urinary infections in females in $85 \%$ [19].

In Bukavu (Democratic Republic of the Congo), Irenge et al. had also found a predominance of urinary infections in females with a sex ratio of 1.73 in favour of female [14]. Several authors [6,7] had found a predominance of UTI in women. It is explained by the passage of organisms from digestive tract to the genital apparatus toward the woman's urinary ways. It is facilitated by the short and large urethra in women. Men are protected from the urinary infections by the distance that separates the anus to the urinary meatus (the masculine urethra is long relatively about 16 centimetres) $[6,7,15]$.

We observed that all age groups were touched by UTI but the age groups which were touched more were those between 16-25 years,
26-35 and 36-45 years respectively with $14.1 \%, 44.2 \%$ and $23.7 \%$. These results are similar to those found by Kamwira in Butembo [15] and Irenge in Bukavu [14]. Akram, in a survey on etiologies and antibiotics resistance of communal UTI at Aligarh Hospital in India, found that the predominant age group was the one between 20-49 years with a prevalence of $51.04 \%$ [20]. The age interval of 16-25 years is the one during which there is adolescence crisis and the mindless sexuality seems to be high during this period. On the other hand, for ages lower than 15 years, the urinary infections would be due to the precariousness of hygiene.

The Gram positive bacteria were more isolated (62.9\%) than the Gram negative bacteria (37.1\%) (Figure 1). These results are different from those of other authors [6,7] who found a predominance of Gram negative bacteria. This would be explained by the fact that bacteria responsible of UTI are variable according to environment [15].

The isolates from urine culture were Staphylococcus aureus (47.0\%), Streptococcus spp (12.0\%), Escherichia coli (10.9\%), Moraxella spp (7.5\%), Bacillus spp (2.3\%), Erwina spp (1.9\%) and Listeria spp (1.7\%) (Table 3). This distribution of bacterial isolates is not the same as the one reported in literature by other authors $[14,15]$. 
Page 6 of 7

Irenge et al. found in their study on the antimicrobial resistance of isolates from urine cultures in South-Kivu, that the most frequent bacteria were Escherichia coli, Klebsiella spp. and Enterobacter spp. [14]. In Butembo, a survey conducted in 2014 on the pathogens responsible of UTI had shown that, the main bacteria were Staphylococcus aureus (57.2\%), Escherischia coli (4.71\%), Streptococcus (4.13\%) and Moraxella (2.73\%) [15]. In Europe, it is reported that, according to their frequency, the following pathogens are responsible for UTI: Escherichia coli, Enterococcus, Pseudomonas aeruginosa, Klebsiella, Proteus mirabilis and the Enterobacter [21]. This difference is due to the variation in the geographical distribution of the germs. This one can even be observed in the same hospital.

Staphylococcus aureus is the most frequently isolates from urine cultures in this study. It would be explained by the fact that the Staphylococcus aureus is ubiquitous germ finding in soil, air and water. It is also commensal germ of skin and the mucous membranes. It can be also found as normal flora in oropharynx and in stools. A third of individuals are carrier of Staphylococcus aureus in their nasal pits. The carelessness of hygiene is a factor that favors the infections bound to this species [22].

All isolated germs and tested for sensitivity showed that they are multi drug resistant (MDR). This study shows that Staphylococcus aureus and Streptococcus spp are resistant to penicillins, carbapenems, aminoglycosides, macrolides and cyclines. E. coli shows resistance to penicillins, carbapenems, cyclines and phenicols. Overall, the most concerning trends in Europe in 2013 were related to the occurrence of resistance in gram-negative bacteria (Escherichia coli, Klebsiella pneumoniae, Pseudomonas aeruginosa and Acinetobacter species) [22]. For Escherichia coli and Klebsiella pneumoniae, a continuous increase in resistance to key antimicrobial groups was noted. A majority of the isolates reported to European Antimicrobial Resistance Surveillance Network (EARS-Net) in 2013 was resistant to at least one of the antimicrobial groups under surveillance, and many of these showed combined resistance to third-generation cephalosporins, fluoroquinolones and aminoglycosides. Over the last four years (2010 to 2013), resistance to third-generation cephalosporins in Klebsiella pneumoniae and Escherichia coli increased significantly at European Union/European Economic Area (EU/EEA) level, as well as in many of the individual Members States. Many of the isolates resistant to thirdgeneration cephalosporins were extended spectrum beta-lactamase (ESBL)-positive and showed resistance to additional antimicrobial groups. In addition, resistance to fluoroquinolones, aminoglycosides and carbapenems, as well as combined resistance to fluoroquinolones, third-generation cephalosporins and aminoglycosides increased significantly at EU/EEA level for Klebsiella pneumoniae, but not for Escherichia coli [22].

In Central Africa, the alarming rate of resistance has been noted for nearly all pathogens. Of special concern were MDR in Shigella and Salmonella spp. as well as the emergence of meticillin-resistant Staphylococcus aureus (MRSA), high-level penicillin-resistant Streptococcus pneumoniae and extended-spectrum B-lactamases (ESBL) among Gram-negative pathogens [23].

According to the Multiple Antibiotics Resistance Indices, all bacterial isolates show a MARI greater than 0.20 (Table 4). This implies that a very large proportion of the bacterial isolates have been exposed to several antibiotics and thus have developed resistance to these antibiotics. Bunduki et al. [24] had already shown the irrational use of antibiotics among student in this area. In that study, antibiotics were used irrationally in $76.1 \%$ for treating UTI and the antibiotics most used were amoxicillin-clavulanic acid (Augmentin), penicillin, amoxicillin, ciprofloxacin, erythromycin, and doxycycline. This may expose bacteria to several antibiotics.

\section{Conclusion}

S. aureus, Streptococcus pneumoniae spp, Escherichia coli, Moraxella spp and Bacillus spp were the most prevalent among the uropathogens investigated. Continuous monitoring of antimicrobial susceptibility pattern of bacterial isolates implicated in UTI is needed seeing the high MARI of all samples isolates. And this should be prior to antibiotic prescription in order to ensure an optimal, desired and cost-effectiveness treatment. However, for empiric treatment of UTIs in Butembo, we suggested ciprofloxacin, cefuroxime and cefotaxime as the first line antibiotics of choice. Further studies have to be done for determining the phenotypic and genotypic resistance characterization of the multidrug resistant pathogens isolated in this study.

\section{Conflicts of Interest Statement}

All authors declare no competing interests regarding the publication of this paper.

\section{Funding}

This study has been funded by the Else-Kröner-Fresenius-Stiftung through the BEBUC Scholarship System.

\section{Acknowledgments}

Authors would like to thank all technicians at the department of microbiology at the Central Laboratory of Research of the "Université Catholique du Graben" for their collaboration during this study.

\section{References}

1. WHO (2015) Global antimicrobial resistance surveillance system. Manual for early implementation, Geneva.

2. WHO (2014) Antimicrobial resistance. Global report on surveillance, Geneva.

3. WHO (2015) Worldwide country situation analysis: response to antimicrobial resistance, Geneva

4. Thriemer K, Katuala Y, Batoko B, Alworonga JP, Devlieger H, et al. (2013) Antibiotic prescribing in D.R. Congo: A knowledge, attitude and practice survey among medical doctors and students. Plos one 8: e55495.5.

5. WHO (2012) The evolving threat of antimicrobial resistance. Options for action, Geneva.

6. Rudramurthy KG, Kumaran R, Geetha RK (2015) Etiology and antimicrobial susceptibility pattern of bacterial agents from urinary tract infection in a tertiary care centre. Int J Sci Stud 2: 125-127.

7. Ahmed I, Sajed M, Sultan A, Murtaza I, Yousaf S. et al. (2015) The erratic antibiotic susceptibility patterns of bacterial pathogens causing urinary tract infections. EXCLI Journal 14: 916-925.

8. Phua DH, Zosel A, Heard K (2009) Dietary supplements and herbal medicine toxicities-when to anticipate them and how to manage them. Int J Emerg med 2: 69-76.

9. Shrestha RK, Rai SK, Khanal LK, Mandal PK (2013) Bacteriological study of neonatal sepsis and antibiotic susceptibility pattern of isolates in Kathmandu, Nepal. Nepal Med Coll J 15: 71-73.

10. Soula A, Bougouma AK, Serme AK (1990) Bacteriological study of urinary tract infections in Bamako: Practical orientation. Medicine of Black Africa Medicine of Black Africa 243-249. 
Citation: Bunduki GK, Kibendelwa ZT, Nzanzu AK (2017) In-vitro Antimicrobial Susceptibility Pattern of Isolates from Urine in Butembo,

11. Preethishree P, Rai R, Kumar V, Pai KBA, Bhat UP (2016) Uropathogens and their antibiotic susceptibility pattern at a tertiary care teaching hospital in Coastal Karnataka, India. Int J Curr Microbiol Appl Sci 5: 23-31.

12. Chowdhury S, Parial R (2015) Antibiotic susceptibility patterns of bacteria among urinary tract infections patients in Chittagong, Bangladesh. SMU Medical Journal 2: 114-126.

13. Rezaee MA (2015) Etiology and antimicrobial susceptibility pattern of pathogenic bacteria in children subjected to UTI. A referral hospitalbased study in Northwest of Iran. Medicine 94: e1606.

14. Irenge LM, Kabego L, Vandenberg O, Chirimwami RB, Gala JL (2014) Antimicrobial resistance in urinary isolates from inpatients and outpatients at a tertiary care hospital in South-Kivu Province (Democratic republic of Congo). BMC Res Notes 7: 374.

15. Kamwira S (2014) Bacteria responsible for urinary tract infections in patients examined at the University Graben Diagnostic Center from 2009 to 2013. Background and initiatives. Interdisciplinary J Graben 12: 243-249.

16. Tenke P, Kovacs B, Johansen $\mathrm{TE}^{\mathrm{B}}$, Matsumoto $\mathrm{T}$, et al. (2008) European and Asian guidelines on management and prevention of catheterassociated urinary tract infections. Int J Antimicrob Agents 31S: S68.

17. Forbes BA, Sahm DF, Weissfeld AS (2007) Bailey and Scott's Diagnostic microbiology, 12th edition. Diag Microbiol 12: 842-55.
18. https://clsi.org/education/webinars/

19. Bentroki AA, Gouri A, Yakhlef A, Touref A, Gueroudj A, et al. (2012) Antibiotic resistance of strains isolated from community acquired urinary tract infections between 2007 and 2011 in Guelma (Algeria). Ann Biol Clin 70: 666-668.

20. Akram M, Shahid M, Khan AU (2007) Etiology and antibiotic resistance patterns of community-acquired urinary tract infections in Jawaharlal Nehru Medical College Hospital Aligarh, India. Ann Clin Microbiol Antimicrob 6: 4 .

21. European Centre for Disease Prevention and Control (2014) Antimicrobial resistance surveillance in Europe 2013. Annual report of the European antimicrobial resistance surveillance network (EARS-Net), Stockholm.

22. Chang S, Sievert DM, Hageman JC, Boulton ML, Tenover FC, et al. (2003) Infection with vancomycin-resistant Staphylococcus aureus containing the vanA resistance gene. N Engl J Med 348:1342-1347.

23. Vlieghe E, Phoba MF, Tamfun JM, Jacobs J (2009) Antibiotic resistance among bacterial pathogens in Central Africa: a review of the published literature between 1955 and 2008. Int J Antimicrob Agents 34: 295-303.

24. Bunduki GK, Mumbere M, Mbahweka FK (2017) Assessment of antibiotic self-medication pattern among university students in Eastern Democratic Republic of the Congo. J Pharma Res Intern 18: 1-7. 